\title{
La investigación en educación ambiental en América Latina: un análisis bibliométrico
}

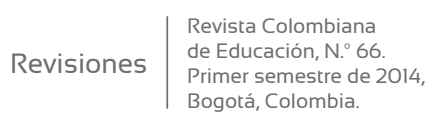

//Research in environmental

education in Latin America:

a bibliometric analysis

\section{//A pesquisa em educação \\ ambiental na América Latina. \\ uma análise bibliométrica}

Iván Felipe Medina Arboleda* Pablo Páramo**
Recibido: 11/02/2014 Evaluado: 18/03/2014 $17 / 03 / 2014$

\section{Profesor de la Universidad Católica de Colombia. Correo electrónico: ifmedina@ucatolica.edu.co \\ Profesor de la Universidad Pedagógica Nacional de Colombia. Correo electrónico: pdeparamo@gmail.com}

\section{Resumen}

El artículo describe y analiza el estado de la investigación en educación ambiental en América Latina a partir de la revisión de los artículos publicados en revistas de educación indexadas en Scielo y Redalyc en el periodo comprendido entre 2000 y 2013. Se construyó una base de datos a partir del año en que fue publicado el artículo, el nombre de la revista, el resumen, el país y la metodología empleada en el estudio. Los resultados permiten observar que, a pesar del gran auge que ha tenido el desarrollo de programas y experiencias en educación ambiental en la región, son pocos los artículos que exploran de manera sistemática su efectividad en la formación de las personas. Se discuten las estrategias metodológicas de las que se valen los investigadores para evaluar el impacto de los programas de educación ambiental en América Latina y la importancia de investigar sobre la evidencia que pueda obtenerse sobre su efectividad.

\section{Abstract}

This article describes and analyzes the state of research in environmental education in Latin America based on the review of articles published in journals of education indexed in SciELO and Redalyc from 2000 to 2013. A database was constructed considering the year in which the article was published, the name of the journal, the abstract, the country and the methodology used in the study. The results evidence that despite the boom of programs and experiences in environmental education in the region, there are few articles that explore systematically their effectiveness in educating people. We discuss the methodological strategies that researchers use to assess the impact of environmental education programs in Latin America and the importance of researching the evidence that can be obtained on their effectiveness.

\section{Resumo}

O artigo descreve e analisa o estado da pesquisa em educação ambiental na América Latina partindo da revisão dos artigos publicados em revistas de educação indexadas em Scielo e Redalyc no período compreendido entre 2000 e 2013. Foi construído um banco de dados incluindo o ano em que foi publicado o artigo, o nome da revista, o resumo, o país e a metodologia usada no estudo. Os resultados permitem observar que apesar do crescimento que tem

\section{Palabras Clave}

Educación ambiental, pedagogía basada en la evidencia, bibliometría.

\section{Keywords}

Environmental education, evidence-based pedagogy, bibliometrics.

\section{Palavras chave}

Educação ambiental, educação baseada na evidência, bibliometría. 
tido o desenvolvimento de programas e experiências de educação ambiental na região, poucos artigos exploram sistematicamente sua efetividade na formação das pessoas. Discutem-se as estratégias metodológicas usadas pelos pesquisadores para avaliar o impacto dos programas de educação ambiental na América Latina e a importância de investigar sobre sua efetividade.

En los últimos años ha aumentado la preocupación de la sociedad por el medio ambiente, gracias, en parte, a los acuerdos establecidos en el último cuarto del siglo XX, como la declaración de Educación Ambiental de Belgrado, la Conferencia de Río de Janeiro de 1992, la conferencia Río + 20, y la declaración del Decenio de las Naciones Unidas de la Educación para el Desarrollo Sostenible (2005-2014). En estos encuentros y cumbres internacionales, se adquirieron múltiples compromisos por parte de los Estados para redefinir sus programas, contemplando la variable ambiental, y desarrollando estrategias efectivas de educación ambiental, como uno de los instrumentos para modificar sustancialmente la relación de la sociedad con la naturaleza.

Entre otros compromisos que han venido afinándose en las diversas conferencias internacionales, están la creación de instituciones y políticas públicas de protección del medio ambiente, la firma de acuerdos internacionales, la preocupación medio ambiental en la agenda multipartidista de las naciones y el trabajo de las ONG ecologistas. Estas acciones, junto a la difusión por medios de comunicación de los problemas ambientales, han contribuido a fortalecer los niveles de preocupación de las personas acerca del estado del ambiente en el planeta.

Las disciplinas científicas no han sido ajenas a la preocupación ambiental internacional, aumentándose el estudio, discusión y aproximaciones sistemáticas a la relación entre el comportamiento humano y el estado del ambiente con el propósito de contribuir a la explicación y comprensión de la problemática ambiental y a la búsqueda de soluciones. Para el caso de las ciencias humanas, su interés ha estado en el estudio de la responsabilidad que tienen los sistemas sociales y culturales sobre buena parte de los problemas ambientales, dado que el comportamiento contextualizado socialmente de las personas y su impacto en el medio ambiente (comportamientos ambientalmente relevantes) es una de las variables fundamentales para resolver eficientemente este tipo de problemas, así como para preservar los recursos y prevenir otros problemas ambientales 
en el futuro (Meadows, Meadows y Randers, 1992; Organización de las Naciones Unidas, 2002; Sandoval, 2012), razón por la que el énfasis en el comportamiento de los individuos como aspecto clave del cuidado del medio ambiente se retomó por la declaración final de la cumbre Río + 20 en el siguiente apartado:

\begin{abstract}
Reconocemos que las personas son el elemento central del desarrollo sostenible $y$, a este respecto, nos esforzamos por lograr un mundo que sea justo, equitativo e inclusivo, y nos comprometemos a trabajar juntos para promover el crecimiento económico sostenido e inclusivo, el desarrollo social y la protección del medio ambiente, lo cual redundará en beneficio de todos (ONU, 2012, p. 2).
\end{abstract}

El compromiso para contrarrestar el impacto ciudadano común sobre el ambiente se ha fijado en la educación ambiental como esfuerzo internacional y multisectorial adoptado por los ministerios de educación nacional de la región latinoamericana. En Colombia, se reglamentó con la Política Nacional de Educación Ambiental (2002), documento legal macro que señala los actores, instituciones, roles y metas de la educación ambiental.

Aunque el acuerdo internacional sobre la importancia de la educación ambiental ha sido ampliamente res- paldado, no existe un único acercamiento sobre la forma en que debe aplicarse, ni de las acciones puntuales que se esperan de los actores educativos (Sauvé, 2004). Por el contrario, los países, sectores sociales y actores educativos presentan diferentes metas respecto al propósito final de la educación ambiental, así como los acercamientos didácticos, epistemológicos y económicos que debería tener, y de los indicadores de efectividad pedagógica resultado de su implementación (Sandoval, 2012; Sauvé, 2004).

Para el caso de los países de América Latina, González (2001) presenta una historia de la educación ambiental en la región, caracterizándola por las tensiones de las propuestas pedagógicas de procedencia de países desarrollados plasmadas en las declaraciones internacionales que no se ajustaban a las condiciones locales de pobreza, desigualdad económica y reivindicaciones sociales, lo que derivó en el desarrollo de ajustes de los discursos sobre la Educación Ambiental que la hicieran más afines con las pedagogías propias latinoamericanas; que posteriormente entrarían nuevamente en tensión con el cambio de denominación de educación ambiental por el de educación para el desarrollo sostenible, periodo en el cual aún se encuentra.

También se retoman en este trabajo (González, 2001) las indefiniciones o ambigüedades en lo que respecta al rol del docente y a la 
caracterización del escenario de interacción entre docentesestudiantes y escenarios no formales de educación, que deriva en que dentro del campo convivan acercamientos centrados en temas de conservación exclusivamente, con otras perspectivas de corte popular que implican la transformación de las condiciones socio-económicas de las comunidades.

En otras palabras, aunque la educación ambiental como la dimensión pedagógica de las transformaciones sociales y económicas propuestas multilateralmente para el afrontamiento de las problemáticas ambientales está llamada a responder una gran demanda social, su establecimiento como campo de práctica pedagógica debe revisarse en términos de sus hallazgos, prácticas, discusiones teóricas y métodos como espacio temático de la pedagogía que darán cuenta de las transformaciones del campo en América Latina.

En dicho contexto, es relevante mencionar las reflexiones de Machado, Lourenço y Silva (2000), quienes proponen tres ejes epistemológicos para evaluar la actividad de las ciencias: el vértice de las investigaciones factuales, el desarrollo teórico y las investigaciones conceptuales. El equilibrio entre las actividades propias de cada uno de los ejes investigativos es sinónimo de prácticas científicas que dan indicios de un buen desarrollo de la ciencia que se esté analizando. Si bien este análisis está originalmente concebido para el análisis de cuerpos disciplinares, también puede usarse para la caracterización de dominios específicos o subcampos de interés teórico. Ello no implica que se esté desconociendo que no todos los acercamientos de la educación ambiental en Latinoamérica concuerden en que esta sea analizada desde una perspectiva de ciencia, pero, aun así, el modelo del triángulo epistemológico sirve para caracterizar teóricamente el campo incluso en ausencia de dicha asunción.

Para el caso del análisis puntual, la evaluación de estos tres ejes permitiría caracterizar a la educación ambiental en Latinoamérica, al menos en lo que a sus publicaciones se refiere. En el primer vértice, encontramos las investigaciones sobre los hechos propios de interés de la disciplina, por ejemplo, aquellos trabajos sobre experiencias pedagógicas, evaluaciones de impacto de intervenciones educativas, planteamiento y evaluación de planes educativos, entre otros. En el segundo vértice, encontramos las investigaciones teóricas que plantean el intento de explicación o comprensión que permitan dar cuenta de los hechos 
registrados en las investigaciones factuales, es decir, son las teorías sobre la educación que están a la base de la explicación del porqué de los hallazgos en las investigaciones factuales del primer vértice. En el tercer vértice, las investigaciones conceptuales sobre las teorías, su significados, alcances, su inteligibilidad y sus dominios sensibles, para el caso, el estudio de las teorías que explican los fenómenos, las revisiones que develan las tensiones del campo, las críticas a los sistemas teóricos, las revisiones de los alcances y limitaciones de los mismos (Machado, Lourenço y Silva, 2000).

El triángulo epistémico en un campo del saber debería mantener el equilibrio entre las clases de investigaciones para no resultar en un colapso del campo de acción; cuando alguno de los vértices se extiende, el triángulo pierde consistencia. Por ejemplo, un exceso de investigación conceptual y teórica dirige la disciplina a discusiones vacías e insorteables sobre conceptos que no tienen ningún asidero en la investigación factual, mientras que un exceso en investigación factual lleva a un conjunto de datos desorganizados, sin un hilo conductor y a la falacia de considerar que la acumulación de registros es en sí misma la labor científica y que la prueba lógica estadística es sinónimo de prueba de validez (Machado, Lourenço y Silva, 2000).

Para el caso de las ciencias, los análisis bibliométricos y las revisio- nes sistemáticas (entre otras herramientas metodológicas) permiten caracterizar en términos intradisciplinares y sociológicos el estado de un campo de conocimiento (Machado, Lourenço y Silva, 2000, para ver un ejemplo) y revisar el grado de acercamiento a las principales demandas sociales que se hacen (Schlinger, 2004).

Un ejemplo de revisión del campo de la educación ambiental para el caso particular de la educación ambiental en el contexto angloparlante es el trabajo de Chawla y Derr (2012), quienes revisaron los trabajos publicados en el área en idioma inglés, con principal interés en aquellos que evaluaban directamente los efectos de las intervenciones pedagógicas en la promoción de comportamientos ambientales (aumento del comportamiento proambiental, disminución de comportamientos que afectan negativamente al medio ambiente), suponiendo que uno de los focos principales de cualquier acción educativa es el cambio en el comportamiento. En su trabajo, subrayan los siguientes aspectos como característicos del campo de estudios en el área:

a. En general, pocos estudios evalúan los efectos sobre el comportamiento de los programas educativos llevados a cabo (menos del $10 \%$ ), quizás relacionados con el costo de las investigaciones, centrándose en el autorreporte y la medida 
de actitudes como indicadores de cambio, con la carga importante de poca predicción que el reporte de dichos aspectos reportan en la investigación (para una revisión, véase Gifford y Sussman, 2012).

b. Los estudios en educación ambiental han privilegiado el formato de clase magistral sin prácticas, lo cual resulta preocupante, teniendo en cuenta que en esta área no solo se presentan aspectos teóricos, sino que también debe establecerse un puente entre lo teórico ambiental, el comportamiento individual y grupal, sus consecuencias y el mantenimiento del comportamiento. Este puente difícilmente se establece con un formato de clase tradicional, en el cual las acciones de los individuos no son modeladas, contextualizadas, ni redundan directamente en el contexto de los individuos.

Para el caso de Latinoamerica, no se encuentran artículos de revisión a partir de estudios bibliométricos, razón por la cual este estudio tiene como objetivo caracterizar, por una parte, la dinámica teórica y metodológica del campo de la educación ambiental publicada en las revistas de educación y, por otra parte, caracterizar la evaluación de la efectividad de los programas de educación ambiental, evaluación de los efectos de las acciones educativas sobre el comportamiento de los participantes.

\section{Método}

\section{Procedimiento}

La ubicación de los artículos de investigación se hizo consultando la Red de Revistas Científicas de América Latina y el Caribe, España y Portugal (Redalyc) y la Librería Científica Electrónica en Línea (Scielo, por el acrónimo en inglés) con los sistemas de información y referenciación Redalyc, teniendo como criterios de ubicación los siguientes aspectos:

- Filtro por publicación: se localizaron exclusivamente publicaciones del área de educación.

- Filtro por palabra clave: se localizaron los artículos que tuviesen como palabra clave educación ambiental. 
- Filtro por temática: se localizaron artículos que abordaran directamente el tema de la educación ambiental, ya fuera como reflexión, ensayo, propuesta, revisión documental, implementación, entre otros.

\section{Resultados}

Después de una depuración de los artículos a partir de 85 artículos, la base de datos quedó constituida por 77 . Los datos se distribuyeron entre la cantidad de artículos por año (tabla 1), por país (tabla 2), por tipo de artículo (tabla 3), por nombre de la revista (tabla 4) y por el método empleado en cada estudio publicado en las revistas (tabla 5).

Tabla 1. Producción por año en revistas de educación con la palabra clave "educación ambiental".

\begin{tabular}{|c|c|}
\hline Año & Número de artículos \\
\hline 2001 & 1 \\
\hline 2002 & 1 \\
\hline 2005 & 2 \\
\hline 2006 & 6 \\
\hline 2007 & 5 \\
\hline 2008 & 12 \\
\hline 2009 & 10 \\
\hline 2010 & 7 \\
\hline 2011 & 12 \\
\hline 2012 & 16 \\
\hline 2013 & 5 \\
\hline
\end{tabular}

En la producción por país identificado (tabla 2), se destacan como los países con mayor productividad
Venezuela, Colombia y México. Este hallazgo debe matizarse teniendo en cuenta que hay un mayor acervo de revistas en español en los portales que en portugués.

\section{Tabla 2. Producción por país en revistas de educación con la palabra clave educa- ción ambiental.}

\begin{tabular}{|c|c|}
\hline País & Artículos \\
\hline México & 14 \\
\hline Colombia & 16 \\
\hline Costa Rica & 6 \\
\hline Venezuela & 26 \\
\hline Brasil & 11 \\
\hline Argentina & 2 \\
\hline Chile & 1 \\
\hline Total & 76 \\
\hline
\end{tabular}

En cuanto al tipo de producción que se encuentra en los portales, la mayor parte de los trabajos se agrupan en la categoría de estudios empíricos, y posteriormente los siguen artículos de tipo teórico, ya sean reflexivos, propuestas pedagógicas sin aplicación actual y revisiones de trabajos previamente publicados (tabla 3).

Tabla 3. Producción por tipo de artículo en revistas de educación con la palabra clave "educación ambiental".

\begin{tabular}{|c|c|}
\hline Tipo de artículos & Número de Artículos \\
\hline Ensayo & 16 \\
\hline Reseña & 2 \\
\hline Empírico & 38 \\
\hline Revisión & 9 \\
\hline Propuesta & 7 \\
\hline Total & 72 \\
\hline
\end{tabular}


En cuanto a las revistas que publican más en el tema de educación ambiental y se encuentran clasificadas como revistas de educación, se encuentran las siguientes: Luna Azul (Colombia) y Educere (Venezuela) (tabla 4). En el primer caso, Luna Azul es una revista especializada en el tema de educación ambiental, para el caso de Educere, si bien no es una revista especializada, concentra gran parte del trabajo ambiental de los educadores de dicho país, por ello, estas dos revistas están a la base de la alta productividad en el campo de estos dos países. Un análisis ulterior podría desarrollarse en términos de la internacionalidad de las revistas (cantidad de autores de diferentes países distintos al de la publicación).

Tabla 4. Nombre de las revistas en que se publican artículos de educación $y$ ambiental.

\begin{tabular}{|l|c|}
\hline \multicolumn{1}{|c|}{ Nombre de la publicación } & Número de Artículos \\
\hline Investigación y Posgrado & 5 \\
\hline Revista de Pedagogía & 2 \\
\hline Revista de Investigación Educativa & 3 \\
\hline Revista Brasilera de Educación & 2 \\
\hline Revista Electrónica Actualidades en Investigación & 6 \\
\hline Revista Electrónica Educare & 2 \\
\hline Movimiento & 1 \\
\hline CPU-e Revista de Investigación en Educación & 2 \\
\hline Ciencia de Educacao & 1 \\
\hline Educere & 10 \\
\hline Universidad, Ciencia y tecnología & 1 \\
\hline Revista Mexicana de Investigación Educativa & 5 \\
\hline Entramado & 1 \\
\hline Luna azul & 12 \\
\hline Magisterio & 1 \\
\hline Linhas Criticas & 3 \\
\hline Omnia & 1 \\
\hline Praxis Educativa & 1 \\
\hline Pérfiles Educativos & 3 \\
\hline Sapiens. Revista Universitaria de Investigación & 3 \\
\hline Paradigma & 1 \\
\hline Educación y Educadores & 1 \\
\hline
\end{tabular}


En lo que respecta a los métodos de investigación que caracterizan el campo de la investigación en educación ambiental, los estudios descriptivos y de carácter de investigación acción son predominantes en el campo. En contraste, los estudios orientados desde perspectivas cuasiexperimentales o experimentales son la minoría.

Tabla 5. Método empleado en las publicaciones empíricas de educación ambiental.

\begin{tabular}{|l|c|}
\hline \multicolumn{1}{|c|}{ Método empleado } & Artículos \\
\hline No aplica & 4 \\
\hline Cualitativo & 3 \\
\hline Descriptivo & 10 \\
\hline Investigación acción & 4 \\
\hline Investigación de campo & 6 \\
\hline Cuasiexperimental & 1 \\
\hline Investigación documental & 5 \\
\hline Investigación exploratoria & 3 \\
\hline
\end{tabular}

\section{Análisis temático de los resultados}

Al revisar las temáticas que se abordan en la investigación sobre educación ambiental, los estudios se pudieron clasificar en cuatro grupos principales: (a) los de carácter descriptivo, (b) los que buscan introducir la educación ambiental en el ámbito de la educación superior, (c) los que evalúan estrategias didácticas o programas específicos sobre educación ambiental y (d) los que se valen del concepto de representaciones sociales para estudiar asi- mismo las representaciones que tienen las personas sobre el ambiente.

Los estudios descriptivos de tipo empírico, que constituyen la mayor parte de lo registrado al hacer la exploración en las bases de datos, se basan en la aplicación de cuestionarios orientados a identificar las principales preocupaciones ambientales y la disposición de estudiantes a colaborar con la protección del medio ambiente $y$, en consecuencia, la importancia de brindar educación ambiental con el fin de concienciar a la población de las amenazas a los recursos naturales (González et al., 2009). Algunos de estos estudios hacen un aporte de tipo metodológico al proponer instrumentos para evaluar las actitudes o el conocimiento que se tiene sobre el estado del ambiente.

Se estudian desde esta perspectiva las preferencias hacia los animales, las exhibiciones, tipo zoológico, y las actividades que allí se realizan con el fin de dar orientaciones para una adecuada gestión educativa ambiental (Moncada et al., 2002), o con la intención de realizar diagnósticos de conocimientos y actitudes con el propósito de hacer propuestas de inserción de la dimensión ambiental en los currículos (Olave de Amigo, 2006) y proponer lineamientos para orientar la educación ambiental para la sustentabilidad de la fauna (González, Moncada y Aranguren, 2009; Moncada y Romero, 2008).

Uno de estos estudios orientados al diagnóstico analiza el estado 
que guarda la educación ambiental en el nivel medio superior mediante el estudio de caso del municipio de Campeche (IsaacMárquez et al., 2011). La investigación, de carácter exploratorio, combina métodos cuantitativos y cualitativos para diagnosticar el grado de cultura ambiental de los estudiantes de preparatoria y el tipo de educación ambiental que reciben. Los resultados indican que los estudiantes poseen un nivel de cultura ambiental bajo y carecen de los conocimientos y habilidades necesarias para realizar cambios ambientalmente favorables en sus estilos de vida. Aunque manifiestan interés por la temática ambiental, tanto el contexto institucional como el bajo nivel de habilitación de los maestros operan como factores que desincentivan a los alumnos. Los resultados permiten identificar ventanas de oportunidad para la educación ambiental a la luz de las actitudes positivas de los alumnos, su interés por aprender prácticas sustentables y la importancia de la escuela como fuente de información ambiental.

Otro ejemplo característico de los estudios diagnóstico es el que se realiza para investigar cuáles eran las concepciones y expectativas de los profesores de enseñanza primaria y secundaria en relación con el trabajo con proyectos de educación ambiental (Queiroz y Carniatto, 2011). Para tal propósito, los profesores respondieron a una encuesta. Los resultados analizados indicaron que los profesores poseen grandes anhelos y dificultades clasificados de la siguiente manera: a) falta de integración entre los profesores de las diferentes disciplinas que torna inviable la práctica de la interdisciplinariedad; b) sobrecarga de actividades que genera falta de tiempo para compartir conocimientos, indagar y reflexionar sobre su práctica docente y c) ausencia de un equipo pedagógico que les auxilie en el desarrollo de los trabajos propuestos.

Varios estudios se han desarrollado con el propósito de incorporar la educación ambiental en la educación superior. La investigación de Valero (2008) y Sosa et al. (2010), por ejemplo, tienen como propósito desarrollar modelos didácticos para la enseñanza de la educación ambiental a nivel de educación superior y diagnosticar el grado de cultura ambiental de los estudiantes. También se han desarrollado con el propósito de reflexionar sobre los principios que han de orientar la educación ambiental en la escuela. De ahí el interés de algunas instituciones de enseñanza superior en insertar la temática de la educación ambiental en 
la formación de maestros (García de Meir y Ruiz Morón, 2008). Con el objetivo de determinar el grado de inclusión de la dimensión ambiental en el quehacer educativo de nivel superior en Manizales (Sepúlveda, 2009), se analizaron los aspectos concernientes a investigación, proyección y formación de profesionales en las cinco principales universidades de la ciudad mediante un estudio descriptivo con componente cualitativo y cuantitativo.

Algunos estudios se centran en estudiar los factores que propician e impiden la participación de los estudiantes en el programa de manejo de residuos como el de la Universidad Autónoma Metropolitana, unidad Azcapotzalco (Prado y Pérez, 2011).

Otras investigaciones se han centrado en temas como los derechos de los animales (Uribe, 2012), documentos que recogen experiencias orientadas a transformar la cultura ambiental de las instituciones universitarias y en la formación profesional del estudiantado (Hernández et al., 2006), además de servir para justificar programas de educación ambiental a nivel de posgrado (García et al., 2008) y elaborar políticas en general para la educación ambiental a nivel universitario (Díaz, Martínez y Flores, 2008).

La manera como los investigadores de la educación ambiental se han aproximado teóricamente a los problemas ambientales ha sido desde las actitudes y las represen- taciones sociales. En particular, varias investigaciones sobre representaciones sociales sobre el medio ambiente en Latinoamérica se recogen en las bases de datos exploradas. A partir de este constructo, la tipología de representaciones naturalistas, antropocéntricas y globalizantes se ha convertido en una referencia clave en investigaciones de educación ambiental (Flores, 2008 y 2010; González y Valdez, 2012).

Pero el mayor interés de las investigaciones en el campo está en la evaluación de propuestas y programas que se valen de diversas aproximaciones didácticas para educar ambientalmente a estudiantes escolares mediante programas de recolección y clasificación de vidrio y plástico, el uso de los anuros como recurso didáctico para la educación ambiental en las unidades con el propósito de diseñar una propuesta que permita mejorar la calidad del proceso de enseñanza y aprendizaje. Se evalúan trabajos puntuales como el Programa Internacional de Educación Ambiental (PIEA), promovido por la Unesco y el PNUMA entre 1975 y 1995 (González y Arias, 2009); el programa de educación ambiental para Comités de Agua Potable Rural (CAPR) en el sur de Chile, ejecutado por el Núcleo Científico Milenio Forecos, el programa de educación ambiental para la cuenca del río Mucujún en Venezuela, programas de educación ambiental para el manejo de desechos hospitalarios en centros 
de salud y la formación de líderes ambientales comunitarios a través de la formación centrada en proyectos (Tovar, 2012). Asimismo, a nivel de ciudad se han hecho evaluaciones sobre los programas que ofrecen las organizaciones gubernamentales y no gubernamentales como la realizada en Manizales, Colombia, para justificar una propuesta de inclusión de la dimensión ambiental en los procesos educativos del municipio.

También se han evaluado materiales educativos como el diseñado para la enseñanza de la conservación del cóndor de los Andes (Vultur gryphus) (Pérez et al., 2010) en el parque nacional natural Los Nevados, localizado en la cordillera Central de Colombia. El material educativo diseñado está dirigido a docentes de los centros educativos, facilitadores y promotores ambientales, guías del parque, turistas y a la comunidad en general. En esta misma dirección, se ha estudiado la importancia que tiene el empleo de las TIC para la formación de gestores ambientales, aunque los resultados apuntan a que no hay consenso entre los participantes sobre la motivación que el uso de las TIC aporta a la formación de profesorado en educación ambiental y que esto se debe a las narrativas que se generan. Aunque para el caso de los jóvenes, uno de los estudios permite concluir que este tipo de estrategias basadas en TIC son eficientes y generan una gran dinámica de aprendizaje en los estudiantes al abrir nuevas posibilidades a la educación ambiental sobre la base del aprendizaje autónomo (Badillo, 2012).

Los programas de educación ambiental son evaluados principalmente mediante estrategias de investigación acción como la realizada con estudiantes que cursaron grado décimo y undécimo en la especialidad de manejo del recurso hídrico a nivel de educación media técnica en la institución educativa técnica Valle de Tenza del municipio de Guateque entre 2007 y 2010 (Pérez, 2011). Otro ejemplo de investigación que se vale de esta aproximación metodológica es la reportada por Vargas y Estupiñán (2012), la cual tuvo como propósito sensibilizar mediante estrategias de educación ambiental a estudiantes de educación media aledaños al páramo de Rabanal, en Samacá (Boyacá, Colombia), sobre la importancia de preservar el ecosistema páramo. La metodología se implementó siguiendo los criterios de la investigación acción, en la que se implementaron estrategias como mapas cognoscitivos para la identificación del conocimiento espacial, sensibilización ambiental mediante ejer- 
cicios de desarrollo sensorial y uso de metáforas, además de experiencias de interacción con el medio ambiente como proceso de conexión con el ecosistema.

Se informa en el estudio que el conocimiento de los estudiantes sobre su ecosistema páramo es mínimo en cuanto a fuentes hídricas, flora, fauna e interacción ejercida desde los habitantes, pero que, mediante la implementación de experiencias sensoriales, salidas de campo y el uso de la metáfora, se logró sensibilizar a los participantes en favor de la protección y mejora del medio ambiente, fortaleciendo sus conocimientos, habilidades y destrezas necesarias para construir valores encaminados a modificar sus patrones de comportamiento. Se concluye que la utilización de todos los sentidos en espacios accesibles amplía las alternativas de exploración y acerca a los estudiantes al contexto real circundante, más allá del conocimiento formal. Finalmente, el estudio de Delgado, Trujillo y Torres (2013), con el que se buscó generar cambios en la percepción con respecto al consumo de agua indirecta en los hogares por medio de la educación ambiental y a través de la metodología investigación acción, con talleres participativos en los que se calculó la hueIla hídrica indirecta por consumo de alimentos. A partir de este cálculo, las comunidades mostraron, de acuerdo con los autores, un cambio sobre el consumo de agua, y de ahí generaron propuestas para mejorar su relación con el recurso y en los líderes, la responsabilidad de transmitir lo aprendido a los pobladores de su vereda.

\section{Discusión}

El análisis bibliométrico de los artículos publicados sobre educación ambiental en revistas indexadas en las bases de datos Scielo y Redalyc permite evidenciar algunas características sobre la investigación que se desarrolla en América Latina en el campo de la educación ambiental en revistas especializadas en educación: su posición diversa, metodológicamente plural, el uso de diferentes tipos de estrategias didácticas y epistemológicamente plural. Este panorama ha sido reportado previamente en el análisis que adelantó Sauvé (2004) para la educación ambiental en un contexto internacional y en el trabajo de González (2001) en su revisión de la historia de la educación ambiental en América Latina.

Aunque buena parte de los estudios son de carácter descriptivo y están encaminados a identificar el grado de conocimiento ambiental, las actitudes o las representaciones que tienen grupos de estudiantes escolares o universitarios sobre el estado del ambiente, la mayoría de estudios buscan poner a prueba distintas estrategias didácticas entre las que se incluyen: el deporte, el arte, programas de reciclaje en las instituciones y experiencias directas en 
el ambiente a través de excursiones, visitas a zoológicos o parques naturales en los distintos niveles de formación, la escuela y la universidad.

La manera de hacerlo es generalmente la investigación acción y, en mucho menor grado, a través de estudios cuasiexperimentales. Por esto mismo, resulta difícil establecer qué cantidad de las investigaciones publicadas permite recoger evidencia suficiente para confrontar las distintas aproximaciones en la búsqueda de la eficacia en la formación sobre educación ambiental. Al predominar los trabajos descriptivos, de campo, documental y tipo ensayo, se desconoce el impacto que tienen las distintas aproximaciones para la enseñanza de la educación ambiental.

Este hallazgo es consistente con lo reportado por Chawla y Derr (2012) en su revisión sobre educación ambiental en inglés, en la cual se encontró que la mayoría de los trabajos no evalúan la efectividad de las intervenciones en términos comportamentales y que aún son predominantes los artículos de carácter descriptivo.

Por otra parte, los principales conceptos en los que se soportan estos estudios son los de actitudes, representaciones sociales, constructivismo y aprendizaje significativo, los cuales son centrales en la teorías pedagógicas y psicológicas, conceptos también frecuentes en el estudio de Chawla y Derr (2012).

En términos de Machado, Lourenço y Silva (2000), habría un buen equilibrio entre la cantidad de artículos publicados en el campo en los tres tipos de investigaciones científicas, empíricas, conceptuales y teóricas, aunque la cantidad de trabajos de investigación en general deja todavía mucho que desear respecto de los trabajos empíricos, puntualmente en lo que tiene que ver con una evaluación de los efectos de las intervenciones en educación ambiental, con miras a acercar el campo de conocimiento con las demandas sociales específicas del campo (Schlinger, 2004).

En términos de unidad de análisis, es importante desarrollar no solo los constructos psicológicos que se supone están a la base del cambio comportamental, sino también considerar la categoría conceptual de comportamientos ambientalmente relevantes, dada su importancia como indicador crucial de éxito de los programas (Chawla y Derr, 2012).

También es importante destacar el interés por incorporar cambios en la estructura curricular de la escuela y de las univer- 
sidades no solo desde el interior de las instituciones, sino en el diseño de la política pública educativa, así como revisiones críticas que señalan las relaciones entre los diferentes actores en la educación ambiental y las relaciones entre el campo de la pedagogía con el sistema económico, que puede ser contrario al interés de promover comportamientos sustentables (Sandoval, 2012).

Finalmente, es importante tener presente que este trabajo se ha centrado en la perspectiva de la educación ambiental en términos de la publicación en revistas de educación exclusivamente. No obstante, un abordaje interdisciplinar más completo implicaría la ampliación del acervo de publicaciones a revisar, incluyendo así revistas de psicología, derecho, economía, entre otras, que dieran información sobre las relaciones de frontera entre los campos. Por otra parte, la inclusión de análisis de mapas de citaciones también podría aportar información referente al grado de integraciónseparación de las diversas disciplinas respecto a este tema de interés científico social.

\section{Referencias bibliográficas}

Badillo, M. (2012). Propuesta de comunicación y educación ambiental a través del Facebook y el uso de narrativas digitales. Revista Entramado, 8(1).
Chawla, L. y Derr, V. (2012). The development of conservation behaviors in childhood and youth. En. S. Clayton. (ed), The Oxford handbook of environmental and conservative psychology (pp. 527-555). Oxford: Oxford University Press.

Delgado S., Trujillo, J. y Torres, M. (2013). La huella hídrica como una estrategia de educación ambiental enfocada a la gestión del recurso hídrico: ejercicio con comunidades rurales de Villavicencio. Luna Azúl, 36, 70-77.

Díaz, E., Martínez, E. y Flores, A. (2008). Formulación de las políticas de educación ambiental en el contexto del desarrollo endógeno, sustentable y humano: un modelo para las Instituciones de Educación Superior en Venezuela. Paradígma (2), 115-134.

Flores, R. (2008). Representaciones sociales del medio ambiente. Perfiles educativos, 30(120), 33-62.

Flores, R. (2010). Medio ambiente y educación ambiental: representaciones sociales de los profesores en formación. Magis: Revista Internacional de Investigación en Educación, 2(4), 8.

García Tovar, M., Carrero de Blanco, A., Carrera Moreira, B., ReboIledo, G., Castro, A. y Pérez, D. (2008). El doctorado de educación ambiental de la Upel 
(ipc-ipmjmsm): por qué, para qué y cómo. Investigación y posgrado, 23(2), 383-410.

García, M. y Ruiz Morón, D. (2008). El debate discursivo modernidad-posmodernidad y la educación ambiental en la escuela contemporánea. Educere, 12(042).

Gifford, R. y Sussman, R. (2012). Environmental attitudes. En. S. Clayton. (ed.), The Oxford handbook of environmental and conservative psychology (pp. 65-80). Oxford: Oxford University Press.

González, E. (2001). Otra lectura a la historia de la educación ambiental en América Latina y el Caribe. Desenvolvimiento e Meio Ambiente, 3, 141-158.

González, E. y Arias, M. (2009). La educación ambiental institucionalizada: actos fallidos y horizontes de posibilidad. Perfiles Educativos, 31(124), 58-68.

González, A., Moncada, J. y Aranguren, J. (2009). Los visitantes del parque zoológico y botánico Bararida, estado Lara: demanda real e implicaciones educativas ambientales. Investigación y posgrado, 24(3), 213-238.

González, B., Sierra, V., Cárdenas, F., Muraira, Y. y Martínez, J. (2009). Percepción ambiental en estudiantes de secundaria. Revista Electrónica Actualidades Investigativas en Educación (3), 1-19.

González, E., y Valdez, R. (2012). Enfoques y sujetos en los estudios sobre representaciones sociales de medio ambiente en tres países de lberoamérica. Revista CPU-e (14), 1-17.

Isaac-Márquez, R., García, O., Spencer, A., Arcipreste, M., Aguilar, M., Isaac-Márquez, A. y Acevedo, L. (2011). Cultura ambiental en estudiantes de bachillerato. Estudio de caso de la educación ambiental en el nivel medio superior de Campeche. Revista Electrónica de Investigación Educativa, 13(2), 84.

Machado, A., Lourenço, O. y Silva, J. (2000). Facts, concepts, and theories: the shape of psychology's epistemic triangle. Behavior and Philosophy, 28, 1-40.

Meadows, D., Meadow, D. y Randers, J. (1992). Más allá de los límites del crecimiento. Madrid: El País Aguilar.

Moncada, J., Aranguren, J., Díaz, E., Del Castillo, M. y Benaya, J. (2002). Implicaciones prácticas de las preferencias de los visitantes del parque zoológico Caricuao. Caracas. Investigación y Posgrado, 17(1), 135-158. 
Moncada, J. y Romero, N. (2008). Los centros de interés en la enseñanza de la educación ambiental a nivel superior. Caso: instituto pedagógico de caracas e instituto pedagógico de Maracay. Educere, 12(041).

Olave de Amigo, R. (2006). Conocimientos y actitudes ante el ambiente para la incorporación de la dimensión ambiental en el pénsum de ingeniería industrial. Universidad, Ciencia y Tecnología, 10(38), 62-66.

Organización de las Naciones Unidas. (2012). El futuro que queremos. Recuperado de https:// rio20.un.org/sites/rio20.un.org/ files/a-conf.216-I-1_spanish. pdf.pdf

Perez, S. (2011). Educación ambiental: estrategia en la enseñanza de contaminación en fuentes hídricas. Luna Azul, 32, 10-14.

Pérez, J., Molano, C., Flórez, J., Rendón, D. y Flórez, G. (2010). Diseño de material educativo para la enseñanza de la conservación del cóndor de los Andes (Vultur gryphus). Luna Azúl, 30, 197-203.

Prado y Pérez, (2011). Participación estudiantil en programas ambientales en instituciones de educación superior. Perfiles Educativos, 33(134), 77-98.

Queiroz Amaral, A. y Carniatto, I. (2011). Concepções sobre projetos de educação ambiental na formação continuada de professores. Revista Electrónica de
Investigación en Educación en Ciencias, 6(1), 113-123.

Rojas, L., Delgado, M., Esquivel, C., Chacón, O., Fallas, J., Segreda, A. y Serrano, E. (2011). La dimensión ambiental en el currículo universitario: un proceso de cambio en la formación profesional. Actualidades Investigativas en Educación.

Sauvé, L. (2004). Una cartografía de corrientes en educación ambiental. En M. Sato e I. Carvalho (org.), A pesquisa em educação ambiental: cartografías de uma identidade narrativa em formação. Porto Alegre: Artmed.

Sandoval, M. (2012) Comportamiento sustentable y educación ambiental: una visión desde las prácticas culturales. Revista Latinoamericana de Psicología, 44(1), 181-196

Sepúlveda Gallego, L. (2009). Una evaluación de los procesos educativo-ambientales de Manizales. Luna Azul (28), 46-56.

Sosa, S., Isaac-Márquez, R., Eastmond, A., Ayala, M. y Arteaga, M. (2010). Educación superior y cultura ambiental en el sureste de México. Universidad y Ciencia, 26(1), 33-49.

Schlinger, H. (2004). Why psychology hasn't kept its promises. The Journal of Mind and Behavior, 25(2), 123-144.

Uribe, J. (2012). Animal rights: moral theory and practice. CPU-e, Revista de Investigación Educativa (14), 167-177. 
Tovar, J. (2012). Fundamentos para la formación de líderes ambientales comunitarios: consideraciones sociológicas, deontológicas, epistemológicas, pedagógicas y didácticas. Luna Azul, 34, 214-239.

Valero, N. (2011). La educación ambiental en las instituciones de educación superior del estado Bolívar, Venezuela. Revista de Pedagogía, 29(85).

Vargas, C. y Estupiñán, M. (2012). Estrategias para la educación ambiental con escolares pobladores del páramo Rabanal (Boyacá). Luna Azul (34), 10-25. 